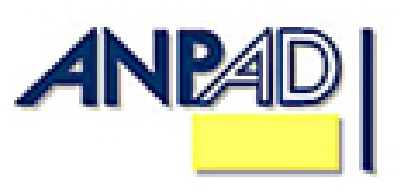

Disponível em http://www.anpad.org.br/rac

RAC, Curitiba, v. 14, n. 3, art. 7, pp. 516-531, Mai./Jun., 2010

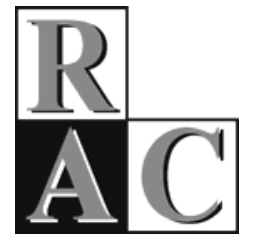

\title{
Estilos de Comportamento de Profissionais de Ciência, Tecnologia e Inovação (C,T\&l)
}

\author{
Professional Behavioral Styles in Science, Technology and Innovation (STI)
}

* Endereço: Roberto Coda

Universidade Municipal de São Caetano do Sul. R. Santo Antonio, 50, Centro, São Caetano do Sul/ SP, 09521160. E-mail: roberto.coda@uscs.edu.br

Copyright (C) 2010 RAC. Todos os direitos, inclusive de tradução, são reservados. É permitido citar parte de artigos sem autorização prévia desde que seja identificada a fonte. 


\title{
RESUMO
}

A partir dos conceitos de Orientação Motivacional e de Estilo de Mobilização, criados com base no pensamento de Erich Fromm sobre personalidade, e à luz de estudos voltados para a gestão de pessoas em ambiente de C,T\&I, este trabalho visa identificar padrões de comportamento no trabalho de profissionais que atuam em organizações de C,T\&I, intencionando, dessa forma, fornecer subsídios para a contratação, desenvolvimento e alocação de profissionais, hoje denominados trabalhadores do conhecimento. Em uma abordagem empíricoanalítica, utiliza-se o Diagnóstico M.A.R.E. para coletar dados de amostra de 643 profissionais de organizações de C,T\&I e os compara com os de outra amostra, composta por 4.981 casos, previamente coletados em organizações de diferentes áreas de atuação e em âmbito nacional. Diferentemente do que ocorre em relação à amostra nacional, os resultados indicam haver predominância, nas organizações alvo do estudo, dos estilos de mobilização Especialista e Competidor, e um decréscimo de representatividade dos estilos de mobilização Mantenedor e Conquistador, sugerindo haver peculiaridades de comportamento e de cultura organizacional para os profissionais e organizações estudados, o que os caracteriza como grupo diferenciado, com padrões comportamentais específicos.

Palavras-chave: estilo comportamental; gestão estratégica de pessoas; ambiente de C,T\&I.

\begin{abstract}
Using the constructs of Motivational Orientation and Mobilization Style based on Erich Fromm's thoughts on personality and considering studies related to people management in STI environments, this paper aims to identify behavioral work patterns of professionals operating in STI organizations as a way to provide insights for the hiring, development and staffing of knowledge workers. The empirical research uses the M.A.R.E. Diagnostic to gather data of a sample of 643 professionals performing research duties mainly in R\&D institutes and compares them to those of a sample composed of 4,981 cases collected nationwide and in organizations of different kinds. Results show predominance of the Mobilization Styles named Specialist and Competitor as well as a reduction in the styles denominated Maintainer and Conqueror, as behavioral work patterns, suggesting the existence of different and specific behaviors and organizational culture for the professionals and organizations involved in the study.
\end{abstract}

Key-words: behavioral style; strategic people management; STI environment. 


\section{INTRODUÇÃO}

\section{Objetivos do Trabalho}

Em ambientes organizacionais, a conscientização e a identificação de padrões ou estilos comportamentais contribuem para o reconhecimento de tendências nas ações dos profissionais, facilitando seu gerenciamento, orientando seu treinamento e desenvolvimento, bem como sua alocação no trabalho por mediação da garantia de um equilíbrio entre preferências individuais e necessidades ou requisitos dos cargos que ocupam.

Este trabalho pretende utilizar uma abordagem concebida especialmente dentro do contexto cultural das organizações brasileiras, a partir de contribuições de especialistas em comportamento humano, para identificar e descrever padrões de ação de profissionais em situações de trabalho, aplicando-a em organizações atuantes na área de C,T\&I.

A contribuição pretende verificar se há estilos ou padrões dominantes de comportamento dos profissionais que estão construindo suas carreiras nessa área, o que poderá vir a fornecer subsídios para a sua contratação, retenção e desenvolvimento, por meio do respeito e consideração dos inerentes pontos fortes e fracos associados ao estilo representativo.

Especificamente, em comparação com os resultados da amostra brasileira, pretende-se verificar se nas organizações de C,T\&I os profissionais apresentam os sete Estilos de Mobilização Brasileiros e se existe predominância de algum deles. Tal confirmação os caracterizaria diferentemente dos respondentes da amostra nacional, entre os quais não há prevalência de padrão de comportamento, contribuindo para maior compreensão das peculiaridades da área e sugerindo características que podem ser utilizadas na incorporação, capacitação e organização de equipes de pesquisa.

\section{GESTÃo de PESSOAS EM AMBIENTE DE C,T\&I}

O pressuposto desse tópico é tecer considerações sobre particularidades da gestão de pessoas em organizações que lidam com ciência, tecnologia e inovação, no que diz respeito a temas tais como motivação, retenção e eficácia no aproveitamento de talentos, bem como buscar subsídios para a identificação de perfis comportamentais profissionais mais presentes nessas instituições e suas respectivas características.

Recente pesquisa teve como objetivo caracterizar o que representa um ambiente de pesquisa favorável, a partir de levantamento realizado com três importantes laboratórios do Departamento de Energia do governo americano. Jordan (2005) identifica, em primeiro lugar, que há surpreendentemente poucos dados empíricos disponíveis para ajudar gestores de institutos de pesquisa na tarefa de atrair e reter trabalhadores eficazes na área de Pesquisa e Desenvolvimento [P\&D]. A maioria das pesquisas sobre atitudes e satisfação desses trabalhadores não contempla os atributos específicos que cientistas e engenheiros consideram ser particularmente importantes para organizações de pesquisa. Esses estudos não consideram, por exemplo, que pesquisadores trabalham menos por dinheiro e mais por glória, isto é, pelo desafio de superar problemas científicos ou técnicos, bem como pela satisfação de exercitar sua criatividade e obter aprovação do seu trabalho da parte dos seus pares. $\mathrm{O}$ estudo aponta que a qualidade dos profissionais de pesquisa é o fator mais importante para a excelência nas atividades de $\mathrm{P} \& \mathrm{D}$. O elenco completo de fatores para retenção e motivação de pesquisadores revelados neste trabalho inclui os seguintes aspectos: visão e estratégia de pesquisa existentes no instituto, investimento em competências futuras, senso de desafio e de entusiasmo, indicadores de sucesso em nível de projeto, estilo de gestão informal e decisivo, reconhecimento e recompensas baseados no mérito, identificação de novas oportunidades, valorização a longo prazo da 
pesquisa fundamental e cuidadosa interpretação dos resultados (Jordan, 2005). A presença, portanto, desses diferentes fatores configura aquilo que, na visão dos pesquisadores consultados, seria o ambiente de pesquisa adequado, uma vez que a presença desses fatores alavancaria a satisfação e o desempenho dos pesquisadores.

Maccoby (2005) considera que expressiva parcela do trabalho do gestor de P\&D é compreender as pessoas com quem trabalha, não somente fazendo uma avaliação de como elas se desempenham da função, mas principalmente tentando prever como irão atuar em novos papéis ou funções. Sugere que, diferentemente do que ocorre nas ciências naturais, a dificuldade apontada encontra-se no fato de que não é possível realizar experimentos controlados e em laboratório com pessoas e, mesmo que fosse possível, o número de variáveis envolvidas seria de tal ordem, que ficaria inviável avaliar todas elas. Por outro lado, indica como saída a utilização de teorias e tipologias psicológicas para o desafio de compreender pessoas nas organizações, até mesmo nas organizações de ciência, tecnologia e inovação.

Embora existam diferentes abordagens e metodologias para a avaliação de traços de personalidade, como, por exemplo, o diagnóstico Myers-Briggs Type Indicator [MBTI], que toma por base a contribuição do psicanalista C. G. Jung, Maccoby (2005) considera particularmente útil, para a compreensão de pessoas e de comportamentos gerenciais no trabalho, as contribuições de Freud e as de seu revisionista, à luz do aspecto cultural, Eric Fromm, que propôs as orientações Receptiva, Acumuladora, Exploradora e Mercantil, como formas de interação do indivíduo, as quais definem sua maneira de ser.

A contribuição de Fromm para estudos posteriores, em especial aos voltados para os estilos de comportamento, centra-se em quatro comportamentos básicos: Aceitar, Tomar, Conservar e Permutar, com seus aspectos positivos e negativos, resultantes da combinação de orientações identificadas com base no processo de assimilação (Fromm, 1990, p. 116; Cf. Ricco, 2004, pp. 31-38). Para ele, todas as orientações se encontram na bagagem humana. Se há o predomínio de qualquer uma delas, é sobretudo em função da peculiaridade da cultura em que o indivíduo vive (Fromm, 1983, p. 74).

Destaca-se também a contribuição do estudo de Beck e Wade (2004), traçando um perfil das personalidades de jovens profissionais que atuam na área técnica e de pesquisa. As características citadas nesse último estudo são especialização (ser competente em algo e saber recuperar-se do fracasso); considerar tudo possível (sempre há uma resposta que é encontrada na base da tentativa e erro); competição como estado natural (não esperar por nada); trabalho em equipe (desde que estruturado de tal modo que cada um tenha seu papel específico); visão global, ambição, preferência por recompensas, baseadas em resultados e desconsideração da liderança.

Sem dúvida, contar com grupo de profissionais técnicos altamente motivados é um dos ingredientes essenciais para aumentar o desempenho inovador necessário, para que a organização continue sobrevivendo no atual ambiente competitivo global (Cf. Mangelsdorf, 2009). Katz (2005), ao aplicar o modelo de enriquecimento do trabalho de Hackman e Oldham (1980) a profissionais que atuam na área de pesquisa e desenvolvimento, aponta que estabelecer uma identidade profissional é o primeiro e crítico passo na direção da construção de uma carreira técnica de sucesso.

É importante também destacar que, ao aplicar o modelo da excelência em negócios ao contexto de dois laboratórios de pesquisa, desenvolvimento e inovação, como forma de descobrir o que significa uma gestão de qualidade em pesquisa, Weggeman e Groeneveld (2005) operacionalizam uma série de recomendações tangíveis, as quais se revelaram úteis para uma administração eficaz das atividades de pesquisa. No âmbito do presente trabalho, é de especial interesse a sugestão de efetuar a mensuração de papéis preferidos ou existentes dentro dos diferentes grupos de pesquisa, identificando aqueles que são mais responsáveis pela geração de idéias técnicas de valia, quais os que questionam sistematicamente as idéias geradas, quais os que conduzem os experimentos mais críticos, quais os que contribuem para a construção de parcerias eficazes, quais os que estabelecem conexões com o negócio, e assim por diante, de modo a permitir e incentivar que cada um possa desempenhar o papel que conhece melhor ou aquele a que melhor se adapta. 
Para Maccoby (2006) ninguém parece ter encontrado a maneira perfeita de gerenciar trabalhadores do conhecimento, especialmente aqueles dos quais é esperada a criação de novos conhecimentos. Em sua visão, quanto mais complexa a natureza do trabalho científico desempenhado pelo pesquisador, mais a produtividade irá depender das qualidades apresentadas pelos diferentes indivíduos que atuam como cientistas. Cita, ainda, resultados encontrados por Davenport (2005) voltados para configurar o perfil dos trabalhadores eficazes do conhecimento. Esse perfil indica que pesquisadores eficazes são aqueles que, em primeiro lugar, são bons aprendizes. Em segundo lugar, conseguem estabelecer redes de relacionamento internas e externas do instituto onde atuam, baseadas não na busca de melhores oportunidades de carreira, mas em oportunidades de aumentar seu conhecimento. Finalmente, considera que os profissionais com desempenho superior são aqueles que conseguem tempo para ler, falar com pessoas, refletir e pensar, adotando como postura pessoal eu sei, eu faço. Este autor também afirma que trabalhadores do conhecimento apresentam melhor desempenho, quando compreendem o ramo de negócios no qual sua organização se insere. Para ele uma organização de P\&D é parte de um sistema sociotécnico, que tem propósito e estratégia empresarial claramente definida. Novamente, citando Davenport (2005), deixa claro que trabalhadores do conhecimento ou pesquisadores, especialmente os mais qualificados, são profissionais difíceis de gerenciar. São pessoas que detestam a burocracia e que criticam mudanças organizacionais emanadas da cúpula da organização. Querem trabalhar numa cultura que contemple a colaboração; a melhor maneira de engajá-los em novas políticas e processos é envolvê-los no desenho de seu próprio trabalho. Por outro lado, podem necessitar de educação voltada para a eficácia educacional, como, por exemplo, saber conduzir uma reunião para obter bons resultados, usar tecnologia pessoal, organizar a informação eficientemente e ensinar aos outros aquilo que sabe. Nesta missão de gerenciar esse tipo de profissional, enfatiza, ainda, a importância, para os líderes de equipes, de entender a personalidade dos pesquisadores da equipe, levando em conta as diferenças individuais por eles apresentadas.

Conduzido por Vojak, Griffin, Price e Perlov (2006), outro estudo, voltado para explorar as características que mais frequentemente aparecem em profissionais técnicos que atuam em pesquisa industrial e que são percebidas como aquelas que mais contribuem para que seja obtido sucesso nas atividades desempenhadas, aponta que o principal traço desses profissionais é incorporar, de modo eficaz, múltiplas tecnologias à estratégia empresarial, identificando inovadoras aplicações para produtos e processos. A amostra utilizada foi de 418 pesquisadores americanos e ingleses e o estudo fornece também considerações a respeito do perfil e das características e traços desses profissionais. Além da reconhecida profundidade e amplitude de seu conhecimento técnico, esse tipo de profissional encontra-se emocionalmente envolvido com o que faz, demonstra grande energia no trabalho, é persistente na busca e realização de objetivos e conta com habilidades de relacionamento interpessoal. Profundo conhecedor do negócio de sua organização, detentor de elevada competência para explorar as oportunidades que seu ambiente lhe oferece e idealista, são as outras características com as quais tais profissionais se apresentam e que, muitas vezes, acabam contribuindo para o sucesso alcançado, mais do que suas próprias competências técnicas.

\section{A LINHA De Pesquisa M.A.R.E. E OS ESTILOS De MobilizaÇÃo Brasileiros}

Iniciada com a construção e validação de um instrumento de coleta de dados, denominado Diagnóstico M.A.R.E. de Orientação Motivacional, a linha de pesquisa M.A.R.E. vem avançando no que concerne ao mapeamento de perfis comportamentais brasileiros, sua identificação e aplicações. Apresentada como uma metodologia nacional desenvolvida para identificar comportamentos nas organizações de trabalho, os estudos baseiam-se nas contribuições psicanalíticas de Erich Fromm sobre a personalidade humana e em teorias sobre liderança e motivação no trabalho (Coda, 2000).

O Diagnóstico M.A.R.E. se baseia em quatro orientações motivacionais, cujas iniciais compõem o seu nome. São elas: Mediadora, Analítica, Receptiva e Empreendedora. A Tabela 1 apresenta uma síntese das principais características de cada uma das orientações motivacionais aqui tomadas como 
referencia e já com as adaptações realizadas para o contexto das organizações de trabalho, bem como a visualização do paralelo estabelecido entre a classificação proposta por Coda (2000) e aquela originariamente desenvolvida por Fromm (1990).

Tabela 1

Principais Características das Orientações M.A.R.E.

\begin{tabular}{|c|c|c|c|}
\hline $\begin{array}{l}\text { Mediadora } \\
\text { Mercantil (Fromm) }\end{array}$ & $\begin{array}{l}\text { Analítica } \\
\text { Acumuladora (Fromm) }\end{array}$ & $\begin{array}{l}\text { Receptiva } \\
\text { Receptiva (Fromm) }\end{array}$ & $\begin{array}{l}\text { Empreendedora } \\
\text { Exploradora (Fromm) }\end{array}$ \\
\hline Orientado para & Orientado para estratégias & Orientado para pessoas & Orientado para resultados \\
\hline $\begin{array}{l}\text { relacionamentos } \\
\text { Busca de harmonia e de }\end{array}$ & $\begin{array}{l}\text { Busca continuidade nas } \\
\text { ações e processos }\end{array}$ & $\begin{array}{l}\text { Identificador dos talentos } \\
\text { das pessoas }\end{array}$ & $\begin{array}{l}\text { Prefere mudanças e } \\
\text { desafios constantes }\end{array}$ \\
\hline $\begin{array}{l}\text { integração entre visões } \\
\text { conflitantes em } \\
\text { situações de trabalho }\end{array}$ & $\begin{array}{l}\text { Orientado para elevados } \\
\text { padrões de qualidade em } \\
\text { tarefas e procedimentos }\end{array}$ & \multirow{2}{*}{$\begin{array}{l}\text { Grande interesse em } \\
\text { desenvolver suas próprias } \\
\text { habilidades e } \\
\text { competências, bem como } \\
\text { aquelas dos demais }\end{array}$} & $\begin{array}{l}\text { Grande capacidade de agir } \\
\text { e obter aquilo que dele é } \\
\text { esperado }\end{array}$ \\
\hline $\begin{array}{l}\text { Compreensão das } \\
\text { necessidades das }\end{array}$ & $\begin{array}{l}\text { Guiado pela lógica e } \\
\text { racionalidade }\end{array}$ & & $\begin{array}{l}\text { Movido por poderosa e } \\
\text { expressiva imaginação }\end{array}$ \\
\hline $\begin{array}{l}\text { pessoas } \\
\text { Hábil para vender novas }\end{array}$ & $\begin{array}{l}\text { Excelente visão de longo- } \\
\text { prazo }\end{array}$ & $\begin{array}{l}\text { Reconhece o valor da } \\
\text { diversidade }\end{array}$ & \multirow[t]{3}{*}{$\begin{array}{l}\text { Explora novos mercados e } \\
\text { oportunidades de negócio }\end{array}$} \\
\hline $\begin{array}{l}\text { Facilidade para atuar em } \\
\text { grupos, naturalmente }\end{array}$ & $\begin{array}{l}\text { Impessoal, objetivo e } \\
\text { sincero }\end{array}$ & $\begin{array}{l}\text { Viabiliza coisas em lugar } \\
\text { de dificultá-las }\end{array}$ & \\
\hline $\begin{array}{l}\text { gregário, sociável e } \\
\text { afetuoso }\end{array}$ & Evita riscos & & \\
\hline
\end{tabular}

Nota. Fonte: elaborado com base em Coda, R. (2000, March). Understanding intrinsic motivation: a research among Brazilian professionals. Proceedings of Annual Research Conference of Academy of Human Resource Development, RaleighDurham, North Carolina, USA, pp. 8-11.

Importante salientar que a abordagem considera que os profissionais no trabalho apresentam todas essas orientações durante o exercício de suas atividades; as diferenças ocorrem na quantidade e ordem de preferência de utilização de cada uma delas.

De acordo com Coda (2000), uma versão preliminar do Diagnóstico foi administrada a 540 gerentes e profissionais técnicos e os dados foram tratados com análise dos fatores comuns e com análise discriminante, o que possibilitou detectar os eventos mais relevantes e as alternativas mais sensíveis para a avaliação de cada uma das orientações. Os resultados desta fase identificaram, então, os 16 eventos ou questões e também as 64 alternativas mais adequadas para avaliar as 8 variáveis resultantes, ou seja, cada uma das 4 orientações motivacionais, tanto em condições normais, quanto em trabalho sobre pressão.

Para melhor compreensão do instrumento, a Tabela 2 apresenta as instruções para o seu uso e o Tabela 3 apresenta fragmentos do seu conteúdo. Uma versão completa do Diagnóstico M.A.R.E. pode ser encontrada em Sápia (2009). 
Tabela 2

\section{Diagnóstico M.A.R.E. - Instruções para Preenchimento}

Para cada uma das questões apresentadas você encontrará uma afirmativa inicial que deve ser complementada pelos 4 finais descritivos que a seguem.

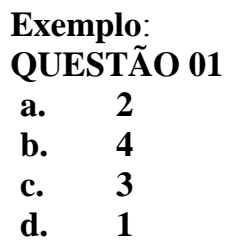

01 GOSTO MUITO DE PESSOAS QUE SÃO:

a. Sinceras, honestas e que inspiram confiança.

b. Rápidas no agir e diretas.

c. Lógicas e ponderadas.

d. flexíveis e sociáveis.

Assim, se a descrição apresentada como complemento da afirmativa proposta que mais se aproxima daquilo que você considera ser verdadeiro sobre você mesmo for aquela contida na letra ' $b$ ', atribua a ela nota 4; se a descrição contida na letra 'c' for quase inteiramente parecida com você, dê-lhe nota 3; se aquela apresentada na letra 'a' for muito pouco parecida com você, atribua-lhe nota 2; e finalmente, se a descrição contida em 'd' não for em nada semelhante à situação que mais favorece a sua motivação no trabalho, coloque nota $\mathbf{1}$ à frente dessa alternativa de descrição, conforme indicado no exemplo acima.

Nota. Fonte: extraído de Miguel, L. F. (2009). Estilos comportamentais dos profissionais da área de relações com investidores. Dissertação de mestrado, Universidade Municipal de São Caetano do Sul, São Caetano do Sul, SP, Brasil, p. 45, e Sápia, T. (2009). Perfis comportamentais e estilos de mobilização de enfermeiros gestores de um serviço de medicina diagnóstica. Dissertação de mestrado, Universidade de São Paulo, São Paulo, SP, Brasil, p. 146.

Tabela 3

Fragmentos do Diagnóstico M.A.R.E.

\begin{tabular}{|c|c|}
\hline Afirmação & Complemento apresentado para escolha \\
\hline $\begin{array}{l}\text { 1. Prefiro realizar meu trabalho em } \\
\text { situações onde posso: }\end{array}$ & $\begin{array}{l}\text { a. Ter independência, autonomia e liberdade para agir. } \\
\text { b. Adaptar e integrar meus conhecimentos às necessidades da } \\
\text { empresa e do grupo com o qual trabalho. } \\
\text { c. Aplicar as técnicas mais adequadas, gerando soluções de } \\
\text { qualidade e dentro dos padrões estabelecidos. } \\
\text { d. Interagir com um grupo que possui grande afinidade. }\end{array}$ \\
\hline $\begin{array}{l}\text { 13. Não gosto de conviver no } \\
\text { trabalho com pessoas que: }\end{array}$ & $\begin{array}{l}\text { a. São dependentes, sem iniciativa e não gostam de ampliar seus } \\
\text { horizontes. } \\
\text { b. São rígidas em seus pontos de vista e insensíveis aos valores e } \\
\text { necessidades do grupo. } \\
\text { c. Deixam-se guiar mais pela intuição e gostam de atuar em } \\
\text { ambiente de constante mudança. } \\
\text { d. São muito individualistas, além de muito críticas em relação ao } \\
\text { grupo. }\end{array}$ \\
\hline
\end{tabular}

Nota. Fonte: Ricco, M. F. F. (2004). Construindo perfis comportamentais em ambiente organizacional: os estilos de mobilização dos gestores brasileiros. Tese de doutorado, Universidade de São Paulo, São Paulo, SP, Brasil, p. 43.

Obtido com amostra significativa, postula-se que um bom valor do indicador estatístico de fidedignidade de um instrumento, ou o coeficiente de fidedignidade de uma escala, dado pelo Alpha de Cronbach, também conhecido como consistência interna do teste, seria acima de 0,70. Assim, o Diagnóstico M.A.R.E. é instrumento confiável, pois apresentou os seguintes coeficientes Alpha: 0,80 para $\mathrm{M}, 0,82$ para A, 0,83 para R, 0,80 para E e um coeficiente Alpha global de 0,91 . 
Essa versão, cujo coeficiente Alpha de Cronbach indica medir o que intenciona, foi aplicada em uma amostra de 1.703 respondentes e validada em 2000, conforme indicam os resultados de diferentes procedimentos estatísticos.

As medidas de posição ou de tendência central — média, moda e mediana — por serem iguais ou similares, indicaram que as ditribuições (global e de cada uma das variáveis) são possivelmente normais. O que foi corroborado pelo teste de Kolmogorov-Smirnov, que apresentou p values $>.05$, indicando aderência das variáveis a uma distribuição normal.

Outro índice, o Fator de Inflação da Variância que, quando maior do que 10, indica multicolinearidade, indicou não haver correlação entre nenhuma das variáveis, pois apresentou $\mathrm{VIF}<1.032$, sugerindo que cada variável mede o que se propõe.

Como as distribuições se mostraram normais e as variáveis não correlacionadas entre si, buscou-se, por meio da Análise de Variância [ANOVA], verificar se os grupos condições normais e sob pressão eram significativamente diferentes. Os resultados dos dois grupos foram diferentes - para condições normais o $\mathrm{F}$ value foi de 404.020 e para o trabalho sob pressão o $\mathrm{F}$ value foi de 124.238 — sugerindo provável existência de diferença estatisticamente significante entre eles. Como a ANOVA foi realizada com um nível de significância de $0,5 \%$ e os valores de $p$ foram menores do que isso - condições normais com $\mathrm{p}<.0001 \mathrm{e}$ trabalho sob pressão com $\mathrm{p}<.000$ — pode-se confiar nos resultados que indicam tratar-se de dois grupos distintos.

A aplicação desse instrumento - Diagnóstico M.A.R.E. - possibilitou a construção de uma base de dados que já ultrapassa 6.500 casos que, por sua vez, contribuiu para que os estudos avançassem de forma a confirmar que as pessoas podem apresentar orientação motivacional diferente, se as condições de trabalhos apresentarem pressão para a sua realização e que tais comportamentos podem ser agrupados formando padrões comportamentais (Coda, Ricco, \& Garcia, 2005a).

Incrementando a base conceitual da linha de pesquisa M.A.R.E., o estudo realizado por Ricco (2004), onde foram identificados estilos gerenciais brasileiros, considera também a relação entre orientação motivacional e escolhas preferenciais de competências de liderança, em contexto organizacional, na busca por resultados. Nesse estudo, como base teórica, são usados os trabalhos realizados por Coda (2000), quanto às orientações motivacionais, e também o trabalho de Hitt (1993), no que concerne às competências de liderança, sendo ambas as abordagens oriundas das contribuições de Fromm (1990). Compuseram a amostra 4.981 casos coletados em 16 diferentes unidades federativas do Brasil em situações de aprendizagem: treinamentos, cursos de pós-graduação e programas in company.

Segundo Ricco (2004), com relação ao aspecto metodológico, o uso de análise de dados multivariada permitiu a identificação dos padrões comportamentais e a classificação posterior dos respondentes; mas os dados foram preparados antes do uso das técnicas propriamente ditas.

$\mathrm{Na}$ preparação dos dados, as estatísticas básicas dos dados brutos indicaram que as variáveis apresentavam tendência de se distribuírem normalmente, pois os indicativos de média, moda e mediana estavam muito próximos entre si, e os indicativos de curtose e de assimetria estavam próximos de 0 (zero), sugerindo que, por se apresentar tão proximamente de uma distribuição normal, o banco de dados utilizado provavelmente possuía uma representatividade real da população. Também pode ser corroborado, segundo os graus de multicolinearidade apresentados (extensão na qual uma variável pode ser explicada pelas outras variáveis na análise), que cada uma das oito variáveis do Diagnóstico M.A.R.E. tem um efeito preditor único (Cf. Ricco, 2004, p. 113). Mesmo assim, para assegurar simetria e ganhar homocedasticidade, normalidade multivariada e linearidade, os dados foram transformados para uma distribuição normal padrão.

A identificação dos padrões ocorreu por meio de análise de conglomerados, sendo conduzida em duas fases: método hierárquico, aplicado às variáveis de pesquisa, para definir o número de grupos existentes e método não-hierárquico, aplicado aos casos, para se definir os grupos. Para a validação 
dos resultados foi extraída uma amostra randômica de aproximadamente $50 \%$ dos casos e realizado o procedimento de agrupamento.

Já para a classificação dos respondentes do Diagnóstico M.A.R.E. nos grupos identificados, utilizouse análise discriminante múltipla, sendo uma amostra randômica e proporcional aos grupos de $80 \%$ dos casos para se encontrar as funções discriminantes; e no restante dos casos, uma amostra de $20 \%$, para confirmar e validar o resultado encontrado. A classificação correta de $96,8 \%$ dos casos da amostra original foi confirmada pela classificação correta de $96,7 \%$ dos casos da amostra de validação (Cf. Ricco, 2004, p. 120).

Em seguida, de acordo com o referencial teórico utilizado, os padrões comportamentais foram descritos e nomeados como os sete Estilos de Mobilização Brasileiros (EMB): Especialista, Colaborador, Conquistador, Mantenedor, Competidor, Negociador e Realizador.

Com base nos Estilos de Mobilização propostos por Ricco (2004), Cestari (2005) investiga as relações entre o perfil comportamental do indivíduo e seu desempenho, diante de uma estratégia motivacional composta pelo estabelecimento de metas, remuneração variável e concessão de promoções por desempenho. O estudo, realizado com 119 gerentes de um banco de varejo, indica que os diferentes estilos apresentam diferença estatisticamente significativa em relação ao cumprimento das metas estabelecidas pela instituição e sugere que os indivíduos com o EMB Conquistador apresentam melhor desempenho em relação aos demais EMB, enquanto os indivíduos de EMB Colaborador e Especialista apresentam pior desempenho, quando comparados aos demais estilos comportamentais.

Dando continuidade à linha de pesquisa, com a utilização do Diagnóstico M.A.R.E. e dos Estilos de Mobilização Brasileiros, outros estudos têm sido realizados, explorando-se, em contextos diferenciados, a questão dos padrões de comportamento e a descrição das competências a eles associados (Cf. Coda, César, \& Faria, 2009; Coda \& Cestari, 2005; Coda, Faria, César, \& Ricco, 2008; Coda, Faria, César, \& Silva, 2008; Coda et al., 2005a, 2005b; Eschberger, 2006; Maciel, 2006; Miguel, 2009; Sápia, 2009; Silva, Rodrigues, Matiy, Matos, \& Rewa, 2005).

\section{Os Estilos de Mobilização Brasileiros (EMB)}

Estilo de Mobilização, como constructo, não existe na literatura sobre comportamento organizacional. A expressão foi criada para atender à necessidade de nomear os padrões comportamentais encontrados por Ricco (2004), resultantes do Diagnóstico M.A.R.E., concebido por Coda (2000). O conceito se refere ao modo peculiar e intencional de agir, nas organizações de trabalho na busca de resultados e é resultante da interação das orientações motivacionais com as escolhas preferenciais de competências de liderança do profissional. Algumas das características dos Estilos de Mobilização Brasileiros estão apresentadas na Tabela 4.

Tabela 4

\section{Características dos Estilos de Mobilização Brasileiros}

\begin{tabular}{|c|c|c|c|c|}
\hline$\sum_{i=1}^{n}$ & 总 & $\begin{array}{c}\text { Principal } \\
\text { Propulsor do } \\
\text { Comportamento }\end{array}$ & $\begin{array}{l}\text { Sentido } \\
\text { da Ação }\end{array}$ & Principais Padrões Comportamentais \\
\hline . & 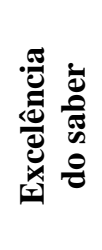 & $\begin{array}{l}\text { Desafios que } \\
\text { exijam ações } \\
\text { cuidadosas e que } \\
\text { produzam } \\
\text { resultados } \\
\text { contínuos }\end{array}$ & $\begin{array}{l}\text { Tornar-se cada } \\
\text { vez melhor } \\
\text { (expoente) em sua } \\
\text { área de atuação }\end{array}$ & $\begin{array}{l}\text { - Age como detentor da verdade e saber } \\
\text { - Realiza seu trabalho de modo persistente e com } \\
\text { cautela, método, proatividadee e independência } \\
\text { - Torna-se social e receptivo se a situação exigir } \\
\text { - Reconhecido como um profissional prático, leal e } \\
\text { cativante }\end{array}$ \\
\hline
\end{tabular}


Tabela 4 (continuação)

\begin{tabular}{|c|c|c|c|c|}
\hline$\sum_{\mid=1}^{\infty}$ & ن⿺辶寸 & $\begin{array}{c}\text { Principal } \\
\text { Propulsor do } \\
\text { Comportamento }\end{array}$ & $\begin{array}{l}\text { Sentido } \\
\text { da Ação }\end{array}$ & Principais Padrões Comportamentais \\
\hline $\begin{array}{l}\frac{\tilde{0}}{0} \\
\frac{0}{0} \\
\frac{0}{0} \\
\dot{0}\end{array}$ & 苞 & $\begin{array}{l}\text { Atividades em } \\
\text { grupo que } \\
\text { possibilitem seu } \\
\text { desenvolvimento } \\
\text { profissional e } \\
\text { pessoal }\end{array}$ & $\begin{array}{l}\text { Busca estabelecer } \\
\text { relacionamentos } \\
\text { que proporcionem } \\
\text { crescimento } \\
\text { mútuo }\end{array}$ & $\begin{array}{l}\text { - Ajuda os outros a encontrar soluções para os } \\
\text { problemas } \\
\text { - Age de modo determinado, mas com jovialidade, e em } \\
\text { resposta às solicitações e demandas (responsivo) } \\
\text { - Apresenta abertura para novas experiências, } \\
\text { aprendizado (conhecimento) e inovações } \\
\text { - Reconhecido como um profissional devotado, } \\
\text { idealista e liberal }\end{array}$ \\
\hline 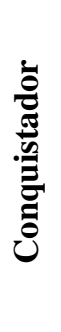 & 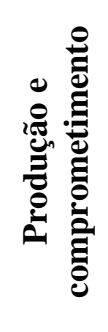 & $\begin{array}{l}\text { Situações de } \\
\text { competição que } \\
\text { resultem em } \\
\text { crescimento } \\
\text { próprio }\end{array}$ & $\begin{array}{l}\text { Conquistar para } \\
\text { ampliar seus } \\
\text { horizontes de } \\
\text { atuação }\end{array}$ & $\begin{array}{l}\text { - Busca resultados contínuos para si e para a organização } \\
\text { - Age de modo autoconfiante, metódico e pró-ativo } \\
\text { - Aprecia desafios constantes para poder demonstrar } \\
\text { sua capacidade atingir metas } \\
\text { - Defende suas ideias com convicção, mas muda } \\
\text { conforme os argumentos } \\
\text { - Reconhecido como um profissional de ação, leal, } \\
\text { cativante e íntegro }\end{array}$ \\
\hline 莺 &  & $\begin{array}{l}\text { Relacionamentos } \\
\text { em que possa } \\
\text { exercer o controle } \\
\text { da situação }\end{array}$ & $\begin{array}{l}\text { Assegurar a } \\
\text { continuidade das } \\
\text { atividades }\end{array}$ & $\begin{array}{l}\text { - Age de modo a assegurar o cumprimento de tarefas } \\
\text { com qualidade } \\
\text { - Busca compreender opiniões e crenças das pessoas, e } \\
\text { identifica talentos } \\
\text { - Realiza seu trabalho de modo persistente, com } \\
\text { cautela, método e em resposta às situações e } \\
\text { demandas (responsivo) } \\
\text { - Reconhecido como um profissional idealista e leal } \\
\text { - Pode-se tornar um líder notável }\end{array}$ \\
\hline ن̃. & 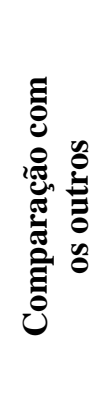 & $\begin{array}{l}\text { Desafios que } \\
\text { dependam de } \\
\text { atuação } \\
\text { competitiva, } \\
\text { decidida e } \\
\text { obstinada }\end{array}$ & $\begin{array}{l}\text { Competir para } \\
\text { vencer }\end{array}$ & $\begin{array}{l}\text { - Naturalmente se compara com os outros, podendo } \\
\text { estabelecer colaboração com outros para atingir } \\
\text { resultados } \\
\text { - Demonstra capacidade para executar o que dele é } \\
\text { esperado, chamando para si a responsabilidade de } \\
\text { resolver os problemas } \\
\text { - Realiza seu trabalho de modo autoconfiante, proativo } \\
\text { e impulsivo } \\
\text { - Reconhecido como um profissional assertivo, } \\
\text { orgulhoso dos próprios feitos e cativante }\end{array}$ \\
\hline 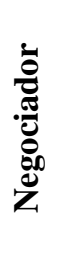 & 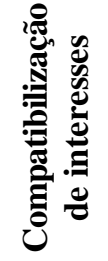 & $\begin{array}{l}\text { Situações que } \\
\text { exijam articulação } \\
\text { para aquisição de } \\
\text { novos valores ou } \\
\text { para manutenção } \\
\text { dos existentes }\end{array}$ & $\begin{array}{l}\text { Convencer o outro } \\
\text { de sua ideia e } \\
\text { obter algo em } \\
\text { troca }\end{array}$ & $\begin{array}{l}\text { - Busca compreensão e equilíbrio entre pontos de vista } \\
\text { conflitantes } \\
\text { - Realiza seu trabalho de forma persitente e inovadora } \\
\text { - Guia-se pela lógica e pela racionalidade } \\
\text { - Reconhecido como um profissional jovial e avançado, } \\
\text { mas precavido }\end{array}$ \\
\hline 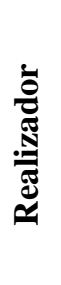 & 㟒 & $\begin{array}{l}\text { Gerenciamento de } \\
\text { situações em que } \\
\text { possa fazer } \\
\text { acontecer }\end{array}$ & $\begin{array}{l}\text { Tornar real o que } \\
\text { foi planejado }\end{array}$ & $\begin{array}{l}\text { - Focado na consecução dos seus objetivos e nos da } \\
\text { organização } \\
\text { - Age de modo determinado, flexível, jovial e proativo } \\
\text { - Prefere mudanças constantes para expor suas idéias, } \\
\text { compatibilizar divergências e realizar o planejado } \\
\text { - Reconhecido como um profissional produtivo e liberal }\end{array}$ \\
\hline
\end{tabular}

Nota. Fonte: elaborado com base em Ricco, M. F. F. (2004). Construindo perfis comportamentais em ambiente organizacional: os estilos de mobilização dos gestores brasileiros. Tese de doutorado, Universidade de São Paulo, São Paulo, SP, Brasil, pp. 130-149. 


\section{Metodologia da Pesquisa}

Realizado com propósito exploratório, este estudo adota uma abordagem empiricoanalítica, uma vez que é marcadamente quantitativo e apresenta forte preocupação com a relação entre variáveis. Foca organizações criadas com o objetivo de conduzir atividades científicas relacionadas à educação, pesquisa e desenvolvimento em diferentes campos - aeroespacial, certificação aeronáutica, energia nuclear, farmacêutico, comunicação digital e petrolífero - e que apresentam significantes produções científicas, responsáveis por avanços tecnológicos para o Brasil em seus respectivos domínios.

Com relação aos dados utilizados, os da amostra brasileira são os apresentados por Ricco (2004). Já aqueles relativos aos profissionais da área de C,T\&I, referem-se a 643 profissionais de organizações de C,T\&I, predominantemente situadas no Estado de São Paulo.

A coleta dos dados, tanto da amostra nacional quanto da amostra de C,T\&I, ocorreu por meio do Diagnóstico M.A.R.E. em programas de capacitação, incluindo-se aqueles de desenvolvimento e treinamento in company.

$\mathrm{Na}$ análise dos dados, no que concerne à identificação dos EMB, faz-se uso de análise multivariada de dados, em especial das sete equações discriminantes, conforme desenvolvido por Ricco (2004). Para a comparação entre as duas amostras, utiliza-se apenas estatística não-paramétrica.

\section{Resultados}

Depois de aplicar o Diagnóstico M.A.R.E. em uma amostra de trabalhadores de organizações de C,T\&I, predominantemente do Estado de São Paulo, foi possível identificar seus diferentes Estilos de Mobilização para fins de comparação, conforme mostra a Tabela 5.

Tabela 5

EMB: comparação entre as organizações de C,T\&I e a amostra brasileira.

\begin{tabular}{cccccc}
\hline \multirow{2}{*}{ Estilo de Mobilização } & \multirow{2}{*}{ Grupo } & \multicolumn{2}{c}{ Organizações de C,T\&I } & \multicolumn{2}{c}{ Amostra Nacional } \\
\cline { 3 - 6 } & & $\#$ & $\mathbf{\%}$ & $\#$ & $\mathbf{\%}$ \\
\hline Especialista & 1 & 153 & 24 & 727 & 15 \\
Colaborador & 2 & 80 & 13 & 774 & 15 \\
Conquistador & 3 & 65 & 10 & 727 & 15 \\
Mantenedor & 4 & 65 & 10 & 717 & 14 \\
Competidor & 5 & 131 & 20 & 655 & 13 \\
Negociador & 6 & 77 & 12 & 696 & 14 \\
Realizador & 7 & 72 & 11 & 684 & 14 \\
\hline Total & - & 643 & 100 & 4981 & 100 \\
\hline
\end{tabular}

Embora todos os EMB apresentados na amostra nacional tenham sido encontrados na amostra das organizações de C,T\&I, a distribuição dos casos da amostra nacional não apresenta diferenças significativas, diferentemente do que ocorre nas organizações de C,T\&I, uma vez que nelas os EMB não se distribuem de forma equitativa. A maior representação - estilo Especialista com $24 \%$ - é mais do que o dobro da representação de menor percentagem, 10\%, apresentada pelos estilos Conquistador e Mantenedor. 
Os dados da Tabela 5 destacam a existência de dois EMB dominantes apresentados pelos trabalhadores de organizações de C,T\&I. Esses dois EMB já representam $44 \%$ dos pesquisados, sendo 24\% de Especialistas (1) e 20\% de Competidores (5). Da mesma forma, salientam-se os Conquistadores (3) e Mantenedores (4), pela pouca representatividade, $10 \%$ cada estilo.

Sem levar em consideração a não significância estatística da diferença entre os EMB da amostra nacional, quanto à sua distribuição, pois as porcentagens foram muito próximas (13\% para Competidores, $14 \%$ para Negociadores e para Realizadores e 15\% para Especialistas, para Colaboradores e para Conquistadores), ao se comparar os resultados das duas amostras (Tabela 5 e Figura 1), há uma inversão na representatividade dos EMB 2 (Colaborador - 13\%) e 3 (Conquistador - 10\%), que apresentam as maiores porcentagens da amostra nacional e algumas das menores da amostra Organizações de C,T\&I, e também do EMB 5 (Competidor - 20\%) que de estilo menos representativo na amostra nacional aparece como dominante nas organizações de C,T\&I. Já o Estilo 1 (Especialista) se mostra dominante na amostra de organizações de C,T\&I (24\%) e equilibrado com os demais na amostra nacional, embora seja um daqueles com maior percentual (15\%). Assim, o fato de haver mais Especialistas e Competidores e menos Conquistadores e Mantenedores na área de C,T\&I parece caracterizar mais o estilo do trabalhador desta área, podendo, até mesmo, sugerir um componente específico na construção da cultura nesse tipo de organização e que se pode revelar associado ao principal traço de identificação do ambiente de C,T\&I.

Os dados da Tabela 5 estão plotados em matriz de dispersão, conforme mostra a Figura 1, permitindo a sua visualização gráfica, na qual é possível identificar os EMB que estão acima e os que estão abaixo da média, que curiosamente é igual para as duas amostras (média amostra nacional = $14,29 \%$ e média organizações de $\mathrm{C}, \mathrm{T} \& \mathrm{I}=14,29 \%$ ), sendo, todavia, os desvios-padrão muito diferentes. Enquanto para a amostra nacional o valor é de $\sigma=5,50$, para a amostra de organizações de C,T\&I esse valor é $\sigma=0,76$.

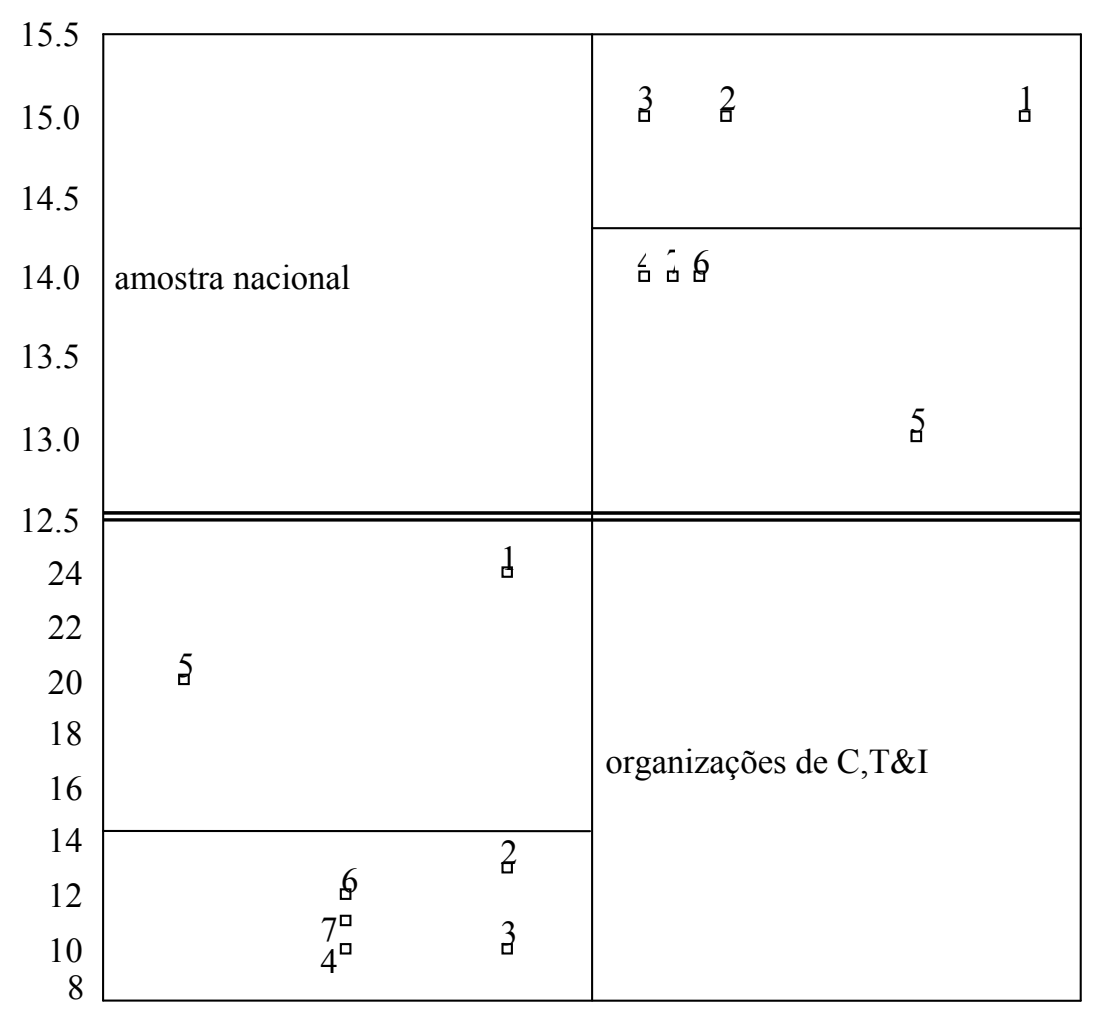

Figura 1: EMB: Matriz de Distribuição das Amostras Nacional e de C,T\&I

A linha horizontal indica os valores médios das duas amostras 


\section{DISCUSSÃo}

No que diz respeito à análise dos objetivos assumidos, o presente estudo confirmou que as organizações de C,T\&I apresentam os mesmos Estilos de Mobilização presentes na amostra brasileira, uma vez que todos os sete diferentes estilos de mobilização foram encontrados nas organizações de ciência, tecnologia e inovação que fizeram parte da coleta de dados. Já a distribuição percentual dos Estilos de Mobilização encontrados nas organizações de C,T\&I, não é a mesma encontrada na amostra brasileira. Na amostra nacional há homogeneidade entre os estilos; mas, no ambiente organizacional de ciência, tecnologia e inovação, observou-se uma predominância dos estilos Especialista e Competidor nos profissionais, revelando, portanto, a existência de estilos de comportamentos profissional dominantes.

Embora não seja possível tomar essa predominância como indicativo de que o estilo Especialista e o estilo Competidor sejam ideais para profissionais que atuam nesse tipo de organização, mesmo porque se trata praticamente de uma amostra regional, os resultados indicam, sem dúvida, que a área de C,T\&I representa uma oportunidade natural de carreira para esses profissionais, já que não só a natureza dessa área ou das organizações voltadas para C,T\&I, mas principalmente a natureza do trabalho e das atividades por eles desempenhadas nelas mostram compatibilidade com as características dominantes de ambos os referidos estilos, promovendo, assim, um ajustamento entre aquilo que é favorecido como via de ação pelo profissional e aquilo que se espera que este execute eficazmente na organização.

Além da evidente maior presença dos estilos Especialista e Competidor, já discutida, há o fato, não menos expressivo, da menor presença dos estilos Conquistador e Mantenedor. Dentre os sete estilos existentes, esses são os dois estilos mais atrelados à existência na organização de um trabalho bem organizado e estruturado, contrariamente aos estilos Especialista e Competidor. Para obter realização profissional, os conquistadores e mantenedores valorizam a liderança e voltam-se, em primeiro lugar, para o atingimento de objetivos e metas organizacionais; comportamentos desejáveis em muitos ramos de negócio; porém não são tão enfatizados pelos chamados trabalhadores do conhecimento que buscam, primeiramente, aumentar seus saberes e obter inovações, pautando-se sempre pelo esmero e qualidade técnica em suas decisões.

Comparando os resultados dessa pesquisa com aqueles de outros estudos e com as considerações de outros estudiosos e pesquisadores do tema gestão de pessoas em organizações de pesquisa, é possível estabelecer um primeiro paralelo com aquilo que é considerado a identidade profissional do empregado técnico. Uma primeira fonte de comparação é o trabalho de Beck e Wade (2004), que revela como características do profissional de pesquisa, entre outras, a especialização, o trabalho em equipe com papéis bem definidos, a competição como estado natural, a visão de que tudo é possível, a preferência por recompensas baseadas em resultados e o desprezo pela função de liderança. Todas são típicas do estilo Especialista que busca o domínio de técnicas específicas associadas ao seu trabalho, ou então a realização de aspectos particulares de sua profissão, como forma de obtenção de qualidade e de excelência em tudo o que faz. Também são compatíveis com o estilo Competidor, a começar pela revelação da constante comparação como um estado de ânimo valorizado por esse profissional. Além disso, a desconsideração da liderança pode ser explicada pela forma independente de agir do Especialista e pela característica do estilo Competidor de chamar para si a comparação com os demais, mesmo com aqueles que estão formalmente nas posições de comando das atividades a serem realizadas como meio de busca de desafios constantes e crescentes.

Os resultados obtidos nessa pesquisa são também compatíveis com as considerações de Davenport (2005; citado por Maccoby, 2006) sobre as características e qualidades dos profissionais do conhecimento. Segundo esse autor, o profissional técnico busca oportunidades de carreira que atendam ao seu desejo de aumentar continuamente seu estoque de conhecimentos. Este ponto revela uma interface com o estilo Competidor, sempre conectado com a identificação de novas oportunidades. Volta-se também para o estabelecimento de desafios constantes que, no contexto das organizações de 
C,T\&I, acaba traduzindo-se por uma vontade de aprender sempre mais. No que se refere ao estilo de mobilização Especialista, o ponto de convergência está associado ao tipo de postura identificado por Davenport (2005) como presente nos trabalhadores do conhecimento, que alicerça sua possibilidade de atuação no domínio das técnicas e saberes associados ao seu ramo profissional. Como fazer, se não sei? Este tipo de preocupação, embora possa ser encontrado também em profissionais que apresentam outros estilos de mobilização, de acordo com as bases teóricas que permitiram a formação dos estilos, é muito mais próximo do padrão Especialista.

Um último ponto de contato, entre o referencial teórico do estudo e os resultados obtidos, é fornecido pelo trabalho de Vojak et al. (2006), segundo o qual o principal traço dos pesquisadores de sucesso é incorporar múltiplas técnicas à estratégia empresarial, identificando aplicações para produtos e processos como meio de fomentar a inovação. Nesse enfoque, é possível depreender uma necessidade de combinar ou garantir a presença tanto do estilo Especialista como a do estilo Competidor na execução das atividades de pesquisa, para que a consecução dos objetivos e o cumprimento do papel da própria instituição fiquem facilitados.

Concluindo este trabalho, torna-se importante ressaltar a importância de conhecer os estilos de mobilização na gestão de trabalhadores, visando poder aproveitar, com maior confiabilidade, as características da personalidade e sua contribuição para os resultados da organização, no mesmo tempo em que é imprescindível lembrar que saber lidar com pessoas representa não somente uma das principais tarefas do gestor de organizações contemporâneas, mas também uma qualidade que o aproxima do seu papel de líder e de motivador de equipes de trabalho. Esforços voltados para a identificação de estilos de mobilização, enquanto manifestações de preferências comportamentais e da motivação intrínseca das pessoas trabalhando em organizações, deverão contribuir para o desafio de respeitar diferenças individuais no trabalho em lugar de simplesmente pretender mudar deliberadamente os trabalhadores. Com base nisso, os estilos de mobilização podem também ser vistos como a base para uma política de gestão de pessoas verdadeiramente voltada para o aproveitamento e alocação do potencial humano, identificando competências nem sempre tão aparentes e dirigindo a atenção para a permanente necessidade de desenvolvimento e de melhoria.

\section{Artigo recebido em 26.01.2009. Aprovado em 16.06.2009.}

\section{REFERÊNCIAS BIBLIOGRÁFICAS}

Beck, J. C., \& Wade, M. (2004). Got game: how the gamer generation is reshaping business. Boston: Harvard Business School Press.

Cestari, R. V. (2005). A relação entre perfis comportamentais e o desempenho eficaz em sistemas motivacionais voltados para a geração de resultados: um estudo em agências bancárias. Dissertação de mestrado, Centro Universitário Álvares Penteado, São Paulo, SP, Brasil.

Coda, R. (2000, March). Understanding intrinsic motivation: a research among Brazilian professionals. Proceedings of Annual Research Conference of Academy of Human Resource Development, Raleigh-Durham, North Carolina, USA.

Coda, R., Cesar, A. M. R. V. C., \& Faria, A. C. (2009). The Old and the new accountant style in Brazil. Proceedings of European Accounting Association, Tampere, Finlândia, 32.

Coda, R., \& Cestari, R. V. (2005). A maximização do desempenho no trabalho como resultante de adequação entre estratégia motivacional e perfil de mobilização de gerentes brasileiros. Proceedings of International Conference of Iberoamerican Academy of Management, Lisboa, Portugal, 4. 
Coda, R., Faria, A. C., César, A. M. R. V. C. \& Ricco, M. F. F. (2008, setembro). Um novo contador? Identificando estilos comportamentais na profissão contábil. Anais do Encontro Nacional da Associação Nacional de Pós-graduação e Pesquisa em Administração, Rio de Janeiro, RJ, Brasil, 32.

Coda, R., Faria, A. C., César, A. M. R. V. C., \& Silva, D. (2008). Behavioral styles in the accounting profession: a study among brazilian graduates. Proceedings of Asian Pacific Conference on International Accounting Issues, Paris, França, 20.

Coda, R., Ricco, M. F. F., \& Garcia, M. N. (2005a, November). Measuring intangible assets: leadership and motivational patterns of behavior in organizations. Proceedings of Asian Pacific Conference on International Accounting Issues, Wellington, New Zealand, 17.

Coda, R., Ricco, M. F. F., \& Garcia, M. N. (2005b, outubro). Motivational orientations and leadership styles in Brazilian organizations. Programa de la Asamblea Anual del Consejo Latinoamericano de Escuelas de Administración, Santiago, Chile, 40.

Davenport, T. H. (2005). Thinking for a living, how to get better performance and results from knowledge workers. Boston: Harvard Business School Press.

Eschberger, W., Jr. (2006). O impacto do estilo de mobilização do gestor na produtividade de equipes de vendas. Dissertação de mestrado, Centro Universitário Álvares Penteado, São Paulo, SP, Brasil.

Fromm, E. (1983). Análise do homem. Rio de Janeiro: Zahar.

Fromm, E. (1990). Man for himself: an inquiry into the psychology of ethics. New York: Henry Holt.

Hackman, J. R., \& Oldham, G. R. (1980). Work redesign. Reading, MA: Addison-Wesley.

Hitt, W. D. (1993). The model leader: a fully functioning person. Leadership \& Organization Development Journal, 14(7), 4-11.

Jordan, G. B. (2005). What matters to R\&D workers? Research Technology Management, 48(3), 2332.

Katz, R. (2005). Motivating technical professionals today. Research Technology Management, 48(6), 29-27.

Maccoby, M. (2005). Understanding the people you manage. Research Technology Management, $48(3), 58-60$.

Maccoby, M. (2006). Is there a best way to lead scientists and engineers? Research Technology Management, 49(1), 60-61.

Maciel, J. D. (2006). Estilos de mobilização, competências profissionais percebidas e opções de áreas de atuação de futuros administradores. Dissertação de mestrado, Centro Universitário Álvares Penteado, São Paulo, SP, Brasil.

Mangelsdorf, M. E. (2009). Motivated to innovate. MIT Sloan Management Review, 50(3), 24.

Miguel, L. F. (2009). Estilos comportamentais dos profissionais da área de relações com investidores. Dissertação de mestrado, Universidade Municipal de São Caetano do Sul, São Caetano do Sul, SP, Brasil.

Ricco, M. F. F. (2004). Construindo perfis comportamentais em ambiente organizacional: os estilos de mobilização dos gestores brasileiros. Tese de doutorado, Universidade de São Paulo, São Paulo, SP, Brasil. 
Sápia, T. (2009). Perfis comportamentais e estilos de mobilização de enfermeiros gestores de um serviço de medicina diagnóstica. Dissertação de mestrado, Universidade de São Paulo, São Paulo, SP, Brasil.

Silva, A. F., Rodrigues, C. F., Matiy, F. S., Matos, J. R., \& Rewa, T. (2005). Estilos de mobilização de gestores de micro e pequenas empresas: um estudo exploratório. Trabalho de Conclusão de Curso, Universidade Presbiteriana Mackenzie, São Paulo, SP, Brasil.

Vojak, B. A., Griffin, A., Price, R. L., \& Perlov, K. (2006). Characteristics of technical visionaries as perceived by American and British industrial physicists. R\&D Management, 36(1), 17-26.

Weggeman, M. P., \& Groeneveld, M. J. (2005). Applying the business excellence model to a research organization. Research Technology Management, 48(4), 9-13. 